lection and to learn the details that they fail to remember. Shorthand enables the student to secure the necessary abstract in writing, and at the same time to give almost as much attention to the subject as if he took no notes. But for this he must have a thorough mastery over the art. The temptation to the student who writes shorthand is to take down too much. A verbatim or really full report of a single lecture will occupy the whole of the evening in its revision and correction, even if it is not written out; and most students have to hear and master three or four lectures a day. To take a full report is a mistake; a good and terse abstract is what is needed, and, as I say, this can be taken in shorthand without any interference with the needful attention to the subjectmatter; nay, the making of the abstract deepens the impression made by the speaker's words, and the student is actually able to carry away in his mind more of the lecture than if he merely listened to it or made an abstract in longhand. Longhand notes, like a leaden weight, keep the student constantly behind the lecturer, so that he has from time to time to omit in order to catch the speaker up. But the whole value of shorthand depends on the efficiency with which it is written. If the student has to attend to his writing, he cannot attend to the lecture. Hence the oftrepeated lesson that shorthand should be taught, and taught thoroughly, at school, so that the student starts in his pursuit of knowledge well equipped with this most useful weapon. If he has not learned it at school, it will still be well worth while for him to devote the needful time at the outset of his studies. He need not seek to acquire a very rapid speed, but he should be able to write 100 words per minute with the perfect ease of almost automatic action. An hour a day for three months will, in most cases, suffice to give the needful dexterity.

Shorthand is most useful to the student of what are called Arts and Literature. The facility which it gives of making brief abstracts of important facts and opinions is extremely valuable. My own old Latin and Greek text-books are studded with shorthand notes--notes of translation, on grammatical construction, on geography and history. But its value to the student of science is still greater. The well-known maxim of Captain Cuttle should be the motto of every student and investigator, and he who can write shorthand will, as a matter of fact, secure twice as many notes, and notes that are twice as useful, as he who does not write shorthand. "There is not time to make a note of it," is always hampering the longhand writer, but to the writer of shorthand the very meaning of the word "time" is almost changed. Minutes acquire the value that hours have to others.

To the student of medicine these remarks apply with peculiar force. Besides the set lectures that he has to attend, much of his teaching is at the bedside of the patient, or in the room in which out-patients are seen, and of the facts that come under his notice, and the lessons he receives, two-thirds at least are lost unless shorthand enables him to secure them. When he ceases to be a nominal student--for he should never really cease to be one-its value still continues, for in notbing is its advantage more strongly felt than in the process of taking notes of the cases of disease that come under his observation and care; notes which are often necessary, not only for his sake, but for the sake of the patient. Whilst the patient is describing his symptoms, they can be taken down in as full detail as is needful, and all the results of the examination can be recorded in a few minutes. I speak from experience, grateful experience, for I possess shorthand notes taken by myself of not less than twenty thousand cases of disease; of one kind of disease alone $I$ have notes of nearly five thousand cases; and $I$ am sure that the notes would not have been half as numerous, and not more than one-third as useful, had they been taken in longhand. And yet, strange to say, in no profession is shorthand less used, and I have observed with much surprise that I am the only member of the medical profession taking part in this Congress. In scientific work in medicine it is the same story. The facility the art gives in abstracting the work of others (I do not mean stealing), in collating facts, in arranging materials, in preliminary composition, in all but preparation for the press, its value is felt at every turn by those who possess the art. But for all this, shorthand must be well known; the worker must be able to write without thought and to read without effort, and the time spared from other work to acquire the needful dexterity will ultimately be repaid a hundredfold.
[I may anticipate a question that is sure to be asked by some readers of this paper, What system of shorthand is the best for these purposes? There is some difference of opinion as to the system best adapted for very rapid verbatim reporting; but there is a general consensus of opinion among shorthand writers of all systems that, for the miscellaneous uses here indicated, Pitman's phonography or phonetic shorthand is the best. Even for rapid writing it stands among the first, and is employed by many of the swiftest living writers. I have written it for nearly thirty years, and can testify to its excellence. Moreover, it has the advantage that there is a very large amount of literature published in it, including the works of many standard authors, several periodicals, and a dictionary of 50,000 words. Thus the facilities for acquiring the necessary ability to read this system are very great.]

\section{SOME CONSIDERATIONS ON THE PATHO. GENESIS OF DISEASES IN WOMEN.}

\section{By WILLIAM B. NEFTEL, M.D. NEW YORI.}

AT the beginning of my medical career $I$ was in charge of a ward for consumptives (tuberculosis of the lungs) in the large hospital attached to the medical school in St. Petersburg. At that time the absolute incurability of tuberculosis was a firmly established dogma, and any attempt to. cure the affection, or even to arrest its progress, would have brought odium upon the investigator; certainly he would have been considered ignorant of scientific medicine, especially of morbid anatomy and pathological histology. Indeed, since the researches of Rokitansky, the progressively destructive character and the constitutional nature of tuberculosis were well known to every educated physician, and against that generalised destructive process it was thought. medical science and art must always remain powerless. The utmost that could be done for these unfortunate patiente was to alleviate their suffering by narcotics, and to diminish the colliquative excretions, at the same time carefully watching the progress of the disease to its fatal termination. The greatest care and study were devoted to the physical examination of these patients, and the diagnostic details had to be verified or corrected at the inevitable necropsy. It is true that at the post-mortem sometimes a character istic cicatrix would be found in the lungs, thus indicating the healing of a former cavity, or, what was still more suggestive, such cicatrices would be found occasionally in the lunge of persons who died of other diseases, and in whom therefore a spontaneous cure of tuberculosis bad undoubtedly taken place at some previous period of life. But such cases were considered simply as exceptions to the rule. And yet the truly scientific physician knows that there can be no exception in nature, and if there exists only one case of cure of tuberculosis, a similar result can be obtained in any given number of such cases, provided all the conditions are known under which the so-called exceptional case got well.

Entirely under the influence of the prevailing notions with regard to the incurability of tuberculosis, I met a case in private practice which by its peculiarity seemed to throw some light upon the etiology of this disease. In a large family, all the members of which were remarkably strong. and healthy, and in which tuberculosis had never existed for generations, one married daughter, twenty-nine years old and mother of two children, was affected with pulmonary consumption. This patient was endowed apparently with the same robust constitution, and even the same features, as the rest of the family; she was brought up in the same locality and under the same healthy sanitary conditions, and yet was the only member of the large family affected with tuberculosis of the lungs. Her disease was diagnosed by competent physicians, and the diagnosis corroborated a few years later at her death.

This patient was for a time under my observation, and gave a detailed account of her previous history, and even explained the cause to which she attributed the origin of her disease. When a girl she was remarkable for robust health. During several years, while living in a fashionable

1 Read in the Section of Medicine of the International Medical Congress, held at Washington. 
boarding-school, she wore day and night a tightly-laced corset in order to have a small waist, and was admired for her beantiful figure. She continued to wear her corset, even while asleep, after having left school, and gave it up only after marriage. Even whilst at school she became pale, and was often troubled with a dry, hacking cough, which, after marriage, became more troublesome, and at times was accompanied by febrile movements and increased expectoration. After the birth of her first child the symptoms of pulmonary consumption became manifest. The disease progressed slowly, and at the age of thirty-three the patient died in an advanced stage of general emaciation.

This case, by the simplicity of the conditions under which the disease originated and developed, could be compared to the exactness of a pathological experiment on animals Indeed, the patient was in perfect health, and without any predisposition to tuberculosis, likeall the other members of her family, but became tuberculous after a special condition had been introduced-a condition entirely absent in the othersviz, tight lacing. The positive result of this experiment appeared to $\mathrm{me}$ at the time a complete demonstration of the fact that a steady methodical compression of the chest and of the upper portion of the abdomen, continued for a certain length of time, can produce tuberculosis in a perfectly healthy person without hereditary predisposition to the affection. Of course there was no difficulty to find a hypothetical explanation of the fact in accordance with the prewailing theoretical ideas. The compression interfered with the free circulation of blood in the lungs, producing stasis, chronic inflammation, and exudation. The latter, according to the existing blood crasis, could become either organised, or else transformed into pus, tubercles, \&c. I was determined to test this hypothesis by experimenting on animals, when working in Virchow's Pathological Institute in 1860. I will give here only a few examples taken at random from my old notes, as all the other experiments present, almost identical results.

Experiment 2.-May 22nd: I applied a bandage, producing moderate pressure, around the chest and abdomen of a wellnourished black rabbit.--23rd: The rabbit manifested a high degree of dyspnoea, and the conjunctivæ were congested and the urinary secretion exceedingly scanty. - 24th and 25th: The symptoms increased in severity, respiration very frequent and superficial, and conjunctivæ dark-red, forming a thick chemotic wall around the cornea.-26th: The animal was found dead. At the necropsy the internal organs, especially those of the abdomen, presented an intense venous congestion, there was dilatation of the right heart, and arterial anæmir of all the parenchymatous organs.

Experiment 3.--On May 29th a bandage with very slight pressure was applied around the chest and abdomen of a well-nourished white rabbit. The symptoms of dyspnœa, \&c, developed more slowly, but in a few days reached the same climax as in the preceding experiment, and on June 1lth the rahbit was found dead, with the post-mortem appearances as in Experiment 2.

Experiment 11.-June 12th: A bandage producing considerable pressure was tied around a large rabbit.-13th: The animal breathed with great difficulty and the bandage was taken off.-14tb. Bandage reapplied quite loosely.-23rd. The rabbit appeared much emaciated, though the morbid symptoms were not acute, and on the 29th was found dead. At the necropsy there was considerable emaciation of the whole body, with arterial anæmia, dilatation of veins and of right heart; in the lungs nothing unusual.

The fatal result was uniform in all the rabbits experimented upon, even when the bandage of the chest and anterior portion of abdomen produced but a slight pressure. I then tried to entirely exclude pressure, and applied the bandage very loosely. Under this modification the acute symptoms would not appear, and some of the animals iived to the end of the session (August), when they were killed mostly by Claude Bernard's piqure; in some, however, death occurred spontaneously. In all of them the necropsy showed arterial anæmia and venous stasis with dilatation of the veins and of the right heart. Especially the liver was always greatly congested and enlarged, but nothing of special interest could be discovered in the lungs. Only once a generalised pathological process was found, closely resembling, if not identical with, tuberculosis. I tried the same experimental method on dogs in Berlin, and continued it in Dr. Harley's laboratory, University College, London, but with a still less satisfactory result. It was almost impossithe to keep the bandage in position on dogs, unless made of
plaster-of-Paris, and even then the animals would gradually succeed in loosening it to such an extent that a positive effect could not be obtained.

Experiment 8.-May 30th : A plaster-of-Paris bandage was applied rather loosely around the chest and anterior portion of the abdomen of a dog. The animal manifested no morbid symptoms.--June 14th : The bandage became perfectly loose, and had to be reapplied.-July 4th: The dog was found dead. At the necropsy a rather moderate degree of arterial anæmia and venous stasis in the internal organs existed. At the lower end of the spleen was a small infarction of the size of a pea. The small intestines were spasmodically contracted. As this was the only case of spontaneous death in a dog after the application of a bandage, I suspected accidental poisoning in the laboratory.

All the other dogs were killed in various ways at the end of the session, the necropsy showing in those where the bandage was efficiently applied general ancmia and dilatation of the right heart, with venous congestion of the parenchymatous organs, but in no case was there any special affection of the lunge. I finally abandoned these experiments, partly because they did not corroborate my original expectation to produce tuberculosis by compressing the chest, but more especially in consequence of Villemin's researches, ${ }^{2}$ which proved that tuberculosis could always be produced by inoculation with tuberculosis. Villemin's views met with much scepticism at the time, but at present they appear quite prophetic. For instance, the contagiousness and specific nature of the organic poison of tuberculosis multiplying itself in the system. \&c. Villemin thus diverted the entire question into a totally different direction, which ultimately led to the discovery of the bacillus tuberculosis.

On account of the negative result of my experiments with regard to the etiology of tuberculosis, I have not published them, although they offer some positive results in other respects. They demonstrate the deleterious effects of a continuous compression of the chest and abdomen in animals, even when applied in a moderate degree. It produces general arterial anæmia and venous stasis in the parenchymatous organs, especially in the liver and other abdominal organs. As regards the question of tuberculosis, the important result obtained by Villemin was soon threatened to be entirely lost by the subsequent work of numerous investigators who with faulty unscientific methods apparently succeeded in proving that tuberculosis can be produced by inoculation not only with tubercular substance, but with almost any substance, organic or inorganic. Fortunately, the admirable researches of Cohnheim's first experiments, conducted with Fraenkel, which, owing to imperfect methods, had led to the erroneous assumption that tuberculosis was produced by the absorption of inspissated pus, and were later refuted by himself, proved conclusively that tuberculosis is a specific infectious disease caused by a distinct living micro-organism present only ingen uine tubercular substances; but its actual demonstration was left to Robert Koch. The brilliant researches of the latter show beyond any possibility of doubt that the bacillus tuberculosis is the real and only cause of tuberculosis, that without it tuberculosis is impossible, and that inoculation with the pure culture of the bacillus always produces tuberculosis. The effect of Koch's discovery on the profession was overwhelming. Not only the diagnosis of the disease became always positive and easy, but it was generally expected that with the knowledge of the etiology of the disease the prophylaxis and the treatment of tuberculosis would become successful. In this respect, unfortunately, the general expectation has not yet been realised, and matters remain exactly as they were before Koch's discovery; now, as before, the fifth part of the population die from tuberculosis. Moreover, although the bacillus tuberculosis is omnipresent, and hence everyone is equally exposed to the infection, yet only certain persons of predilection, those with a so-called phthisical habitus, or under certain conditions, are liable to become affected with pulmonary consumption. In other words, besides the bacillus a certain predisposition on the part of an individual is requisite in order to become consumptive. Thus we are practically brought back to the same condition of things which existed previously to the disease of the bacillus, inasmuch as the primary factor in the production of the disease is the predisposition. So far we are able to form hypothetical

Cause et Nature de la Tuberculose, \&c. Comptes Rendus, Ixi., 1866. Gazette Hebdom., Nos. $48,49$. 
suppositions as to the nature of this predisposition to phthisis. For instance, a person may be considered predisposed when the respiratory mucous membrane is in a state of chronic irritation, and both the local and general resistance are diminished. The interesting researches of Metschnikoff ${ }^{3}$ and his phagocytic doctrine, lend experimental weight and evidence to this hypothesis.

Again, the so-called phthisical habitus, a peculiar faulty formation of the chest, with weak respiratory muscles and general debility, is justly considered a predisposing cause of pulmonary consumption. Under these circumstances, the Iungs not being thoroughly ventilated, the stagnant air and the slow pulmonary circulation afford a favourable condition for the settlement and proliferation of the bacillus tuberculosis. Perhaps the development of tuberculosis in the case above stated could be explained in a similar way. The prolonged compression of the chest by tight lacing in the perfectly healthy person seriously interfered with the normal pulmonary ventilation and circulation, and thus produced the same conditions which are so favourable for the development of consumption in persons with a congenital habitus phthisicus. I therefore maintain, though merely as a nypothesis, that compression of the chest by tight lacing, and continued for a certain lengtin of time, constitutes a predisposing cause of pulmonary consumption.

As already mentioned, my experiments on animals show that a prolonged compression of the chest and abdomen invariably produces anæmia and venous congestion of the liver and other abdominal and pelvic viscera. Analogous results are frequently met with in human pathology. I often had the opportunity, especially in Berlin, of boing present at the necropsy of women in whom the liver presented the characteristic malformation known as corset liver, and of which some illustrations are given in the classical work of Frerichs on Diseases of the Liver. In accordance with our present state of knowledge, the liver plays an important part in the hæmatopoietic function, and not only in the formation of blood, but also in its destruction. It is evident, therefore, that the distorted shape of the liver produced by tight lacing must necessarily interfere with its normal functions, and also that a continual disturbance and obstruction in the system of the portal vein must act injuriously upon the other abdominal organs. Hence the impairment of blood formation, of circulation, of digestion, and the engorgement of the internal sexual organs, the latter condition being a predisposing cause of obstinate chronic affections of the uterus and ovaries. The so-called corset liver is accompanied by a changed shape of the long framework of the chest, the ribs assuming a wrong direction, in which they become permanently fixed.

For the sake of brevity I will not draw clinical pictures of the morbid conditions induced by tight lacing and improper mode of dressing, for every medical practitioner is acquainted with these daily occurrences. It seems necessary, however, to call the attention of the profession to the following experiments, which have a direct bearing upon the subject under consideration. Schreiber ${ }^{4}$ was able to produce experimentally albuminuria in persons whom he subjected to compression of the chest. After moderately compressing the chest in young and perfectly healthy persons, even during a minute and a half only, the urine always contained albumen in quantities varying from a trace to 2 per cent., according to the length of time the compreseion lasted. The albuminuria continued after the cessation of the pressure from one to thirteen hours, the urine containing occasionally, besides, albumen, hyaline casts, and red and white blood-corpuscles. Schreiber attributed the albuminuria following compression of the chest to stasis in the pulmonary circulation, produced by the diminished respixatory expansion of the lungs, and by diminished negative thoracic pressure. This condition leads secondarily to stasis in the renal bloodvessels, with consecutive alteration of the renal tissues.

There can hardly be a doubt in the mind of the medical profession that women's dress, especially the pressure exercised by it upon the chest and abdomen, must necessarily produce an injurious effect upon their health. Indeed, it would be quite impossible for anyone, unaccustomed from childhood to the female wearing apparel, to bear it for a

3 Metschnikoff : Ueber eine Sprosspilzkrankheit, \&c Ueber den kampf der Phagocytengegen Krankheitsarreger (Virchow's Arcbiv, xevi.). Fin Beitrag zur Phagocytenlehre, \&c. (Virchow's Archiv, cvii.). Also Forschritte der Medicin, \&c.

4 Ueber experimentell am Tenschen $2 u$ eryengende Albuminurie Archiv fur exp. Path. und Pharm.: 1885, iii.; and 1886, iv. single day without great discomfort. Only by a con. stant habit of wearing it a certain tolerance is created owing merely to the fact that the compression gradually changes the natural shape of the internal organs, and even of its bony framework, just in the same way as the feet of the Chinese women become distorted by methodical compression. The last comparison is by no means exaggerated, and it can be truly said that the deformed feet of the Chinese women do less harm to their owners than the distorted viscera of our women. In view of this grave. source of discomfort and disease it is hardly worth mentioning the minor inconveniences of women's dress. Anyone, for instance, who would try to wear several hours daily their high-healed shoes would soon become troubled with backache and general fatigue. I believe a certain kind of headache and facial neuralgia, induced by neuritis, is sometimes caused by exposure of the head, covered only by a fashionable bonnet in a northern winter. That such an uncomfortable mode of dressing unfits women for continuous and often hard work is self-evident. It seems strange that women, even those of great intelligence, do not modify their costume more in accordance with hygienic requirements, comfort, æsthetic taste, and common sense. Female fashions, as we all know, have their headquarters in Paris, where they are invented by a clique of uneducated dressmakers, totally ignorant of hygiene and devoid of rsthetic taste. From this principal centre the fashion instantly spreads to all the European courts and to America, and $800 \mathrm{n}$ reaches the remotest parts of the civilised female world. All must submit to the dictates of fashion, which is the most absolute of despotisms, and against the decision of which, no matter how absurd and injurious, an appeal is impossible.

That a sanitary question of such magnitude effecting injuriously half of the population of civilised nations, and capable of transmitting morbid tendencies to future generations, is of vital importance and deserves immediate attention, will be admitted by every physician. I believe the only competent authority capable of remedying the existing evil is the International Medical Congress. After having tried in vain to influence the opinion of my own patients, among whom were a number of intelligent lady physicians in favour of a healthy reform in the way of dressing, I now take the liberty of proposing to the International Medical Congress to appoint a commission to investigate the whole question of dress for women, and to report at the next meeting of the Congress. Such a commission might consist not only of physicians, but also of pedagogues, of artists, and of cultivated ladies from different countries, and would have in view, besides the health and comfort, the rsthetic taste of the sex, of which beauty and modesty are the distinctive characteristics. The work of the commission could begin with an inquiry into the different national costumes, which might offer some practical hints, as their usefulness has already stood the test of centuries, Above all, however, the commission could take into consideration the dress of the classical antiquity, as the idea of simplicity, grace, and comfort, which perhaps would become again a perfect model, when adapted to the changed requirements of modern life and to the various climatic differences. The inauguration of the much-needed reform in the dress of women by the International Medical Congress assembled in America would have a peculiar significance America has already powerfully contributed to the reform in the household affairs of women by the invention and introduction of the sewing, washing, and other machines utensils, and appliances, which have relieved women of so much tedious work, and have saved time now devoted to physical, intellectual, and social culture.

The Medical Atrendance Organisation Committee will resume its sitting on Tuesday next, when they will consider a circular which they propose sending to the Metropolitan Hospital Committees upon the "desirability of forming a union between the hospitals and provident dispensaries," asking them to appoint representatives to attend a conference on this subject. After this the committee will proceed to consider the scheme which they have prepared "for the medical attendance of the industrial classes in the metropolis." If the proposed conference on the first question can be held in the early part of November, it is hoped that the committe will be ready with a full report in December, when a meeting will be called to consider the whole question. 